ARTICLE

https://doi.org/10.1038/s41467-019-08757-2

\title{
High-speed mechano-active multielectrode array for investigating rapid stretch effects on cardiac tissue
}

\author{
Matthias Imboden (1) 1,2, Etienne de Coulon², Alexandre Poulin', Christian Dellenbach², Samuel Rosset ${ }^{1}$, \\ Herbert Shea ${ }^{1}{ }^{1} \&$ Stephan Rohr ${ }^{2}$
}

Systematic investigations of the effects of mechano-electric coupling (MEC) on cellular cardiac electrophysiology lack experimental systems suitable to subject tissues to in-vivo like strain patterns while simultaneously reporting changes in electrical activation. Here, we describe a self-contained motor-less device (mechano-active multielectrode-array, MaMEA) that permits the assessment of impulse conduction along bioengineered strands of cardiac tissue in response to dynamic strain cycles. The device is based on polydimethylsiloxane (PDMS) cell culture substrates patterned with dielectric actuators (DEAs) and compliant gold ion-implanted extracellular electrodes. The DEAs induce uniaxial stretch and compression in defined regions of the PDMS substrate at selectable amplitudes and with rates up to $18 \mathrm{~s}^{-1}$. Conduction along cardiomyocyte strands was found to depend linearly on static strain according to cable theory while, unexpectedly, being completely independent on strain rates. Parallel operation of multiple MaMEAs provides for systematic high-throughput investigations of MEC during spatially patterned mechanical perturbations mimicking in-vivo conditions.

\footnotetext{
${ }^{1}$ Soft Transducers Laboratory, École Polytechnique Fédérale de Lausanne (EPFL), 2002 Neuchâtel, Switzerland. ${ }^{2}$ Department of Physiology, University of Bern, Bühlplatz 5, 3012 Bern, Switzerland. These authors jointly supervised this work: Herbert Shea, Stephan Rohr. Correspondence and requests for materials should be addressed to M.I. (email: mimboden@bu.edu) or to S.R. (email: rohr@pyl.unibe.ch)
} 
M echanical stimuli of appropriate size and timing disturb the electrical function of the heart through mechanoelectrical coupling (MEC) mechanisms. Drastic examples include sudden cardiac death following impacts to the chest by baseballs or, conversely, resuscitation of patients undergoing life-threatening cardiac tachyarrhythmias by an appropriately administered thump to the chest ${ }^{1}$. Experimental investigations of the mechanisms underlying these phenomena require cardiac tissue to be subjected to strain amplitudes and strain rates that range from those encountered during the normal pump cycle of the heart to those present under pathophysiological conditions while permitting the simultaneous monitoring of electrophysiological parameters of interest. Among these, impulse conduction velocity is especially important because it is sensitive to changes in both passive and active electrical properties of cardiac tissue and, hence, is a broad indicator for strain-induced adverse electrophysiological effects on the heart that contribute to the precipitation of arrhythmias ${ }^{2}$. While available experimental approaches based on stepper motor ${ }^{3}$ or indenter technology ${ }^{4}$ permit stretching of cardiac tissues to physiological levels and beyond $\left(>10-15 \%\right.$; for review cf. refs. $\left.{ }^{5-8}\right)$, strain rates offered by these systems are generally substantially slower ${ }^{3}$ or barely exceed ${ }^{4}$ those encountered under physiological conditions $\left(1-2 \mathrm{~s}^{-1}\right.$, determined from changes in sarcomere lengths in intact hearts $\left.{ }^{9}\right)$. To achieve faster strain rates, we developed an experimental system (i) that is based on self-contained, mechanically active cell culture wells, (ii) that permits the reproducible application of defined levels of strain (of at least 10\%) at predefined strain rates (up to $18 \mathrm{~s}^{-1}$ are shown but the technology has the potential to approach strain rates of $1000 \mathrm{~s}^{-1}{ }^{10}$ ) to cultured excitable cells, and (iii) that allows for continuous determinations of conduction velocities immediately after strain application. Experiments with bioengineered strands of rat ventricular cardiomyocytes revealed that, surprisingly, conduction velocity is independent on strain rates even if rates surpass physiological levels by an order of magnitude.

\section{Results}

Self-contained mechano-active multielectrode array. An overview of the components of the mechano-active multielectrode array (MaMEA) system is presented in Fig. 1 and the operating principle of the dielectric actuator (DEA) is outlined in Supplementary Fig. 1 The modular device is built around a cell culture well whose bottom consists of a pre-stretched polydimethylsiloxane (PDMS) membrane that is sandwiched between two ring-shaped printed circuit boards (PCBs) (Fig. 1a, e). The bottom PCB includes high voltage leads for driving the dielectric elastomer actuators (DEAs), whereas the top PCB serves to route the signals from the extracellular electrodes to the interface contacts. A total of 10 linearly arranged and compliant extracellular electrodes are incorporated into the PDMS membrane using gold ion implantation techniques (Fig. 1b) ${ }^{11}$. The two outermost electrode pairs $\left(\mathrm{Stim}_{\mathrm{Els}}\right)$ are patterned such as to permit an efficient bi-polar electrical stimulation of the tissue. The six interposed electrodes $\left(\operatorname{Rec}_{\mathrm{Els}}\right.$, labeled I-VI) are used for recording extracellular action potentials $\left(\mathrm{AP}_{\mathrm{ECs}}\right.$ ) during propagated activity along the tissue. A thin layer of PDMS electrically insulates the Rec $\mathrm{Fl}_{\mathrm{Fl}}$, with the exception of the center region which is in contact with the excitable tissue. Mechanical strain is

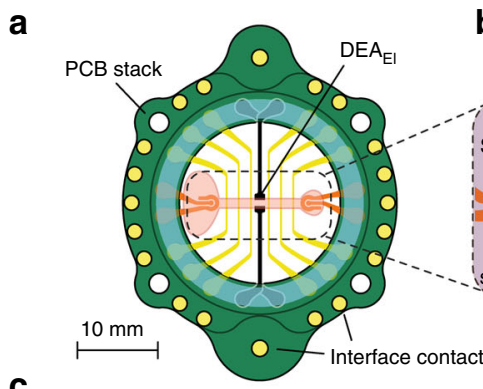

C

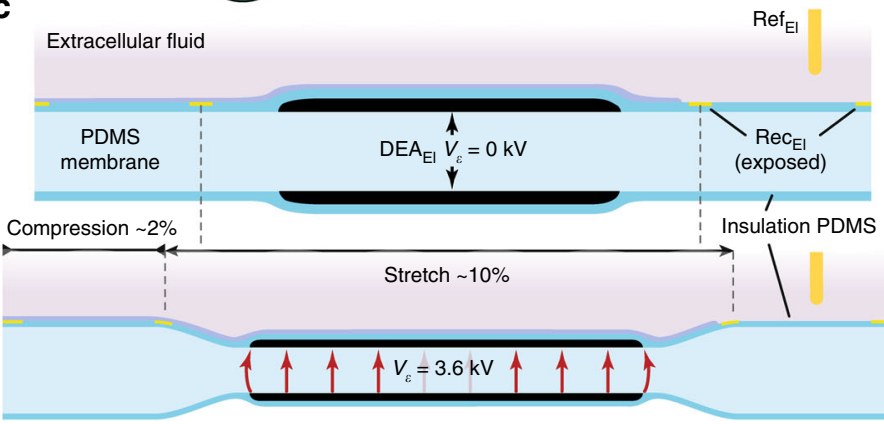

b
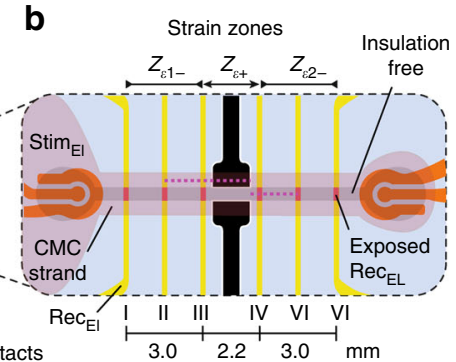

ulation

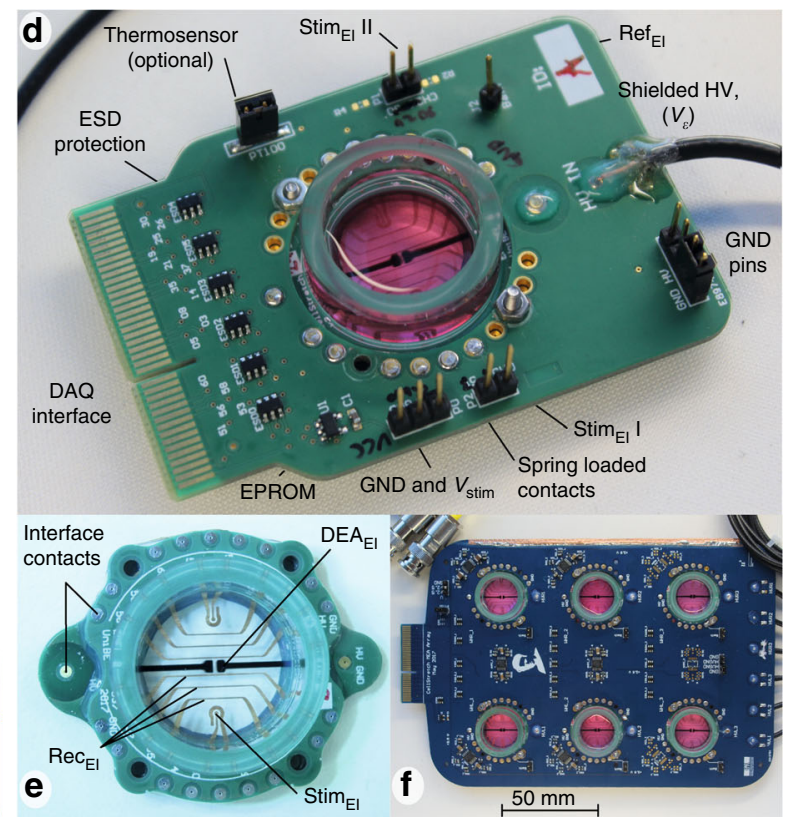

Fig. 1 Mechanically active multielectrode array (MaMEA). a Schematic drawing of the culture well with the bottom being formed by a pre-stretched polydimethylsiloxane (PDMS) membrane that is anchored to two printed circuit board (PCB) frames. The top PCB interfaces with the gold extracellular electrodes (ECEs) that serve to record (recording electrodes, yellow) and stimulate (stimulation electrodes, orange) the patterned cardiac tissue (shape shaded in red). The black structures refer to the ground electrodes (carbon-loaded PDMS) of the dielectric actuator (DEA). The bottom PCB interfaces with the high-voltage DEA electrodes (carbon-loaded PDMS; same shape as ground electrodes). $\mathbf{b}$ Magnified view of the center region of the culture well: application of high voltage to the DEA electrodes results in regions exhibiting positive uniaxial strain $\left(Z_{\varepsilon+;}\right.$ flanked by the two Rec $\mathrm{Cls}_{\mathrm{I}}$ III and IV) and two adjacent zones undergoing negative strain ( $Z_{\varepsilon-}$ with $\operatorname{Rec}_{E \mid s} I-I I I$ and IV-VI, respectively). The dark shaded area within the light-red region (tissue shape) refers to non-insulated regions where cardiomyocytes (CMCs) can make direct contact with the Stimels (indicated in red). c Cross-section of the membrane along the dashed line in $\mathbf{b}$ (not to scale): upon activation of the DEA, the cells in zone $Z_{\varepsilon+}$ are stretched to either side of the center. $\mathbf{d}$ Image of the fully assembled device with the MaMEA mounted on the motherboard that connects to the DAQ. A shielded HV coaxial cable is used to supply the DEA voltage. e Top view of the MaMEA. $\mathbf{f}$ Different implementation of the system consisting of an array of six individually addressable MaMEAs. The motherboard includes signal amplification and conditioning electronics 
generated by two symmetrical DEAs whose design principles were described previously ${ }^{12-16}$ and are outlined in Supplementary Note 1. In short, mechanical actuation is based on the electrostatic force generated by potentials applied between two stretchable carbon electrodes that are separated by a pre-stretched PDMS membrane acting as the dielectric (Fig. 1c). Upon applying an electric field, the incompressible PDMS expands laterally, which induces tensile strain on the membrane between the two sets of DEA electrodes $\left(Z_{\varepsilon+}\right.$ zone) and compressive strain to either side of this central region $\left(Z_{\varepsilon 1-}\right.$ and $Z_{\varepsilon 2-}$ zones; Fig. $\left.1 \mathrm{~b}\right)$. The DEA electrode geometry was chosen such that the membrane between the two DEAs undergoes uniform-uniaxial strain. Because this region is transparent, cells can be microscopically observed in real time during strain application. Further fabrication and actuation details are given in the Methods section and Supplementary Note 2 (Supplementary Fig. 2). Interfacing of the MaMEA with the digital control and acquisition system (DAQ) and the DEA drive signals is accomplished through the motherboard (Fig. 1d) that connects to the cell culture well by means of spring-loaded pins. Because DEA actuation signals can reach up to $5 \mathrm{kV}$, all DAQ connections include electrostatic discharge (ESD) protection to prevent damage to the electronics in the case of high-voltage breakdown of the PDMS membrane. Moreover, Stim ${ }_{\mathrm{Els}}$ are galvanically isolated from the $\operatorname{Rec}_{\mathrm{Fls}}$ in order to reduce the size of stimulation artifacts. While most of the data presented were obtained with single-well devices, a multiwell configuration that enables higher throughput experiments and includes signal amplification and conditioning electronics has been realized as well (Fig. If and Supplementary Note 3, with Supplementary Fig. 3 depicting the functional array configuration of the motherboard with on-board signal conditioning).

The mechanical characteristics of the MaMEA system are illustrated in Fig. 2 with Fig. 2a depicting strain $(\varepsilon)$ development as a function of the applied DEA voltage $\left(V_{\varepsilon}\right)$. While the capacitive force (Maxwell pressure) acting between two fixed electrodes scales with the square of the applied voltage, we observed that $\varepsilon$ in fact scales with $V^{3}$ for strains up to $20 \%$, which is explained by the thinning of the dielectric (PDMS) that accompanies increasing stretch ${ }^{17}$. At $V_{\varepsilon}=3.6 \mathrm{kV}, Z_{\varepsilon+}$ was stretched by $\sim 10 \%$, while both $Z_{\varepsilon-}$ zones $\left(Z_{\varepsilon 1-}\right.$ and $\left.Z_{\varepsilon 2-}\right)$ underwent nearly symmetric compression by $\sim-2 \%$. Strain rates achieved with the MaMEA during maximal stretch $\left(V_{\varepsilon}=3.6 \mathrm{kV}\right)$ were measured using a high-speed camera and are depicted in Fig. 2b. According to the observation that $\varepsilon \sim V^{3}$, constant strain rates were obtained by ramping the DEA voltage as cubic root with respect to time $\left(V_{\varepsilon}\left(0<t<t_{\text {ramp }}\right)=V_{\max } \times \sqrt[3]{\frac{t}{t_{\text {ramp }}}}\right)$ (a discussion of the DEA actuation and strain calibration is presented in Supplementary Note 4). Strain rates applied ranged from of $0.5 \mathrm{~s}$ ${ }^{-1}$ (ramp time, $t_{\text {ramp }}: 200 \mathrm{~ms}$ ) to $18 \mathrm{~s}^{-1}\left(t_{\text {ramp }}: 5 \mathrm{~ms}\right)$.The latter exceeds physiological strain rates $\left(1-2 \mathrm{~s}^{-1}\right)$ and approaches rates associated, for example, with trauma in neurons ${ }^{18-20}$. Strain protocols applied during the biological measurements of this study are shown in Fig. 2c. After a 10-s-long recording of AP propagation under control conditions, preparations were subjected to 10 cycles of identical strains lasting $4.8 \mathrm{~s}$ each $(50 \%$ duty cycle) followed by another $10 \mathrm{~s}$ of baseline recording. Strain amplitudes and $t_{\text {ramp }}$ were systematically changed during successive runs of this protocol that were separated by $>60 \mathrm{~s}$ from each other. Typically, $85 \%$ of total strain was attained during the ramp, thus setting the strain rate. The remaining 15\% developed more slowly due to relaxation processes in the DEA ${ }^{21}$. This "creep" of strain is seen in each cycle and accumulates over the first $\sim 5$ cycles, after which the system reaches steady state. A lesser amount of creep was observed during relaxation. To
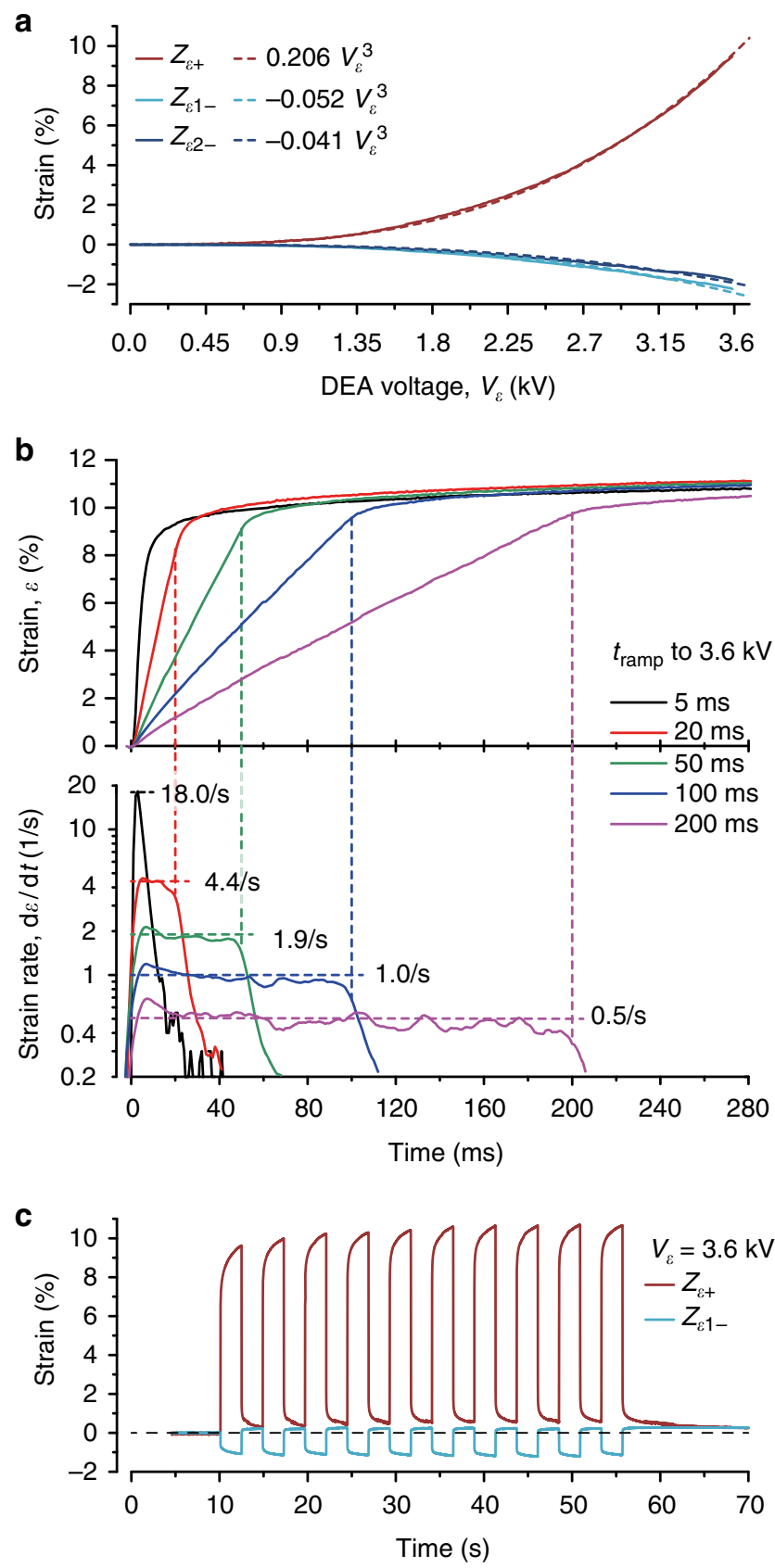

Fig. 2 Mechanical response characteristics of the mechano-active multielectrode array (MaMEA). a Plot of strain in zones $Z_{\varepsilon+,} Z_{\varepsilon 1-}$, and $Z_{\varepsilon 2-}$ as a function of voltage applied to the DEA, $V_{\varepsilon}$. The fit shows a cubic relationship. b Strain (upper panel) and strain rate (lower panel) during the first $280 \mathrm{~ms}$ of linear ramps to $\sim 10 \%$ strain $\left(V_{\varepsilon}=3.6 \mathrm{kV}\right)$. The ramp time ( $t_{\text {ramp }}$ ) is varied from $\sim 5 \mathrm{~ms}$ (step) to $200 \mathrm{~ms}$. The resulting strain rates range from 18 to $0.5 \mathrm{~s}^{-1}$. c Example of strain profiles as used for the experiments. After a 10-s-long baseline recording, the strain is modulated with a period of $4.8 \mathrm{~s}$ for 10 cycles. Protocols are repeated with varying strain amplitudes and ramp times. In the example shown, $t_{\text {ramp }}$ was fixed at $5 \mathrm{~ms}$ (corresponds to a strain rate of $18 \mathrm{~s}^{-1}$ )

account for creep effects, strain development must either be continuously monitored during the experiments or, as done in this study, devices undergo a detailed strain calibration prior or post experiments. Without real-time measurements of strain during the experiments, strain accuracy is limited by creep with an estimated uncertainty of $\sim 5 \%$ of the full strain amplitude (i.e., 
an absolute error of $0.5 \%$ for $10 \%$ strain). A complete set of voltage actuation waveforms and the corresponding predicted strain profiles achieved with the MaMEA is shown in Supplementary Fig. 4.

Assessing electrical activation with compliant electrodes. Primary cultures of neonatal rat ventricular cardiomyocytes were grown on the PDMS membrane as to form a $\sim 16-\mathrm{mm}$-long barbell with the bells covering the ion-implanted Stim Els $_{\text {and }}$ and bar ( $9.7 \mathrm{~mm}$ long, $1.6 \mathrm{~mm}$ wide) covering the non-insulated regions of the $\operatorname{Rec}_{\mathrm{Els}}$ that spanned both the stretch $\left(Z_{\varepsilon+}\right)$ and compression $\left(Z_{\varepsilon_{-}}\right)$regions of the substrate (Figs. 1a, 3a). The cardiomyocyte patterns were generated using lift-off techniques (see Supplementary Note 5 for further details). Cardiomyocyte remained firmly attached to the substrate and to the electrodes during application of strain, that is, $\operatorname{Rec}_{\mathrm{Els}}$ always detected signals from the same group of cardiomyocytes irrespective of the level of strain. The functionality of the gold ion-implanted Stim $_{\text {Els }}$ was tested by assessing the threshold for successful electrical stimulation of the preparations with bi-polar current pulses while systematically varying stimulation amplitude and duration. This resulted in a typical hyperbolic strength-duration curve with the chronaxie amounting to $2.1 \mathrm{~ms}$ at a stimulation strength of 11.1 $\mu \mathrm{A}$ (Fig. 3b). During experiments, cardiomyocyte strands were continuously stimulated at $2.5 \mathrm{~Hz}$ with bi-polar current pulses lasting 2-4 ms at double threshold intensity (typically 20 to 60 $\mu \mathrm{A})$. Gold ion-implanted electrodes also performed well as Rec $\mathrm{Els}_{\mathrm{s}}$ as demonstrated by the example of an $\mathrm{AP}_{\mathrm{EC}}$ recording shown in Fig. 3c. The $\mathrm{AP}_{\mathrm{EC}}$ displayed a typical biphasic shape with an amplitude $\left(V_{\mathrm{pp}}\right)$ of $0.9 \mathrm{mV}$. Further parameters extracted from a Gaussian fit to the first derivative of the $\mathrm{AP}_{\mathrm{EC}}$ included the local activation time, $t_{\mathrm{AT}}$, (fit error of $\sim 6 \mu \mathrm{s}$ ), the maximal downstroke velocity, $\left|\frac{\mathrm{d} V_{\mathrm{ECP}}}{\mathrm{d} t}\right|_{\max }$, and the duration of the downstroke, $t_{\mathrm{DS}}$. $\mathrm{AP}_{\mathrm{EC}}$ recorded from all six $\mathrm{Rec}_{\mathrm{Els}}$ during action potential propagation along a cardiomyocyte cell strand from left to right are shown in Fig. $3 \mathrm{~d}$ with the corresponding plot of $t_{\mathrm{AT}}$ vs. distance depicted in
Fig. 3e (detailed analysis procedures and noise measurements are presented in Supplementary Note 6). As indicated by the linear fit of the $t_{\mathrm{AT}}$ vs. distance data $\left(R^{2}=0.999\right)$, conduction was highly uniform and exhibited an average velocity, $\theta$, of $323.9 \pm$ $0.1 \mathrm{~mm} \mathrm{~s}^{-1}$. The inset in Fig. 3e illustrates that the temporal spread of $t_{\mathrm{AT}}$ measured at $\operatorname{Rec}_{\mathrm{El}} \mathrm{VI}$ during a total of 170 consecutive stimulations at $2.5 \mathrm{~Hz}$ was $<20 \mu \mathrm{s}$. This amounts to $<0.1 \%$ of the overall activation time of the preparation and demonstrates the presence of stable and highly reproducible action potential propagation under control conditions. Supplementary Note 7 characterizes the preparation stability with regards to $t_{\mathrm{AT}}, t_{\mathrm{DS}}, V_{\mathrm{pp}}$, and $\left|\frac{\mathrm{d} V_{\mathrm{ECP}}}{\mathrm{dt}}\right|_{\max }$ without strain modulation.

For the experiments reported in this study, field potentials recorded by the gold ion-implanted electrodes were AC coupled to the amplifiers with a coupling time constant $\left(\tau_{c}\right)$ of $8 \mathrm{~ms}$. While this setting stabilizes signal baselines and thereby permits a precise extraction of local activation times from signals caused by the fast action potential upstroke, the short time constant simultaneously suppresses field potentials arising from slower transmembrane voltage changes associated with repolarization. Supplementary Note 8 demonstrates that, accordingly, an increase of $\tau_{\mathrm{c}}$ to $1600 \mathrm{~ms}$ unveils the repolarization-dependent signal which permits, for example, to assess frequency-dependent changes in action potential duration (APD restitution, see Supplementary Fig. 10). In the same supplementary note, we also show that the MaMEA system is suitable to record electrograms from stem-cell derived human cardiomyocytes (Supplementary Fig. 11). Both of these findings illustrate that the MaMEA system is suitable for cell types different from neonatal rat ventricular cardiomyocytes and that the system may also be used for investigating the effects of strain cycles on repolarization and action potential duration, respectively.

Measuring impulse conduction in strained preparations. To determine the effect of strain on the impulse propagation, preparations were paced at $2.5 \mathrm{~Hz}$ while being subjected to the
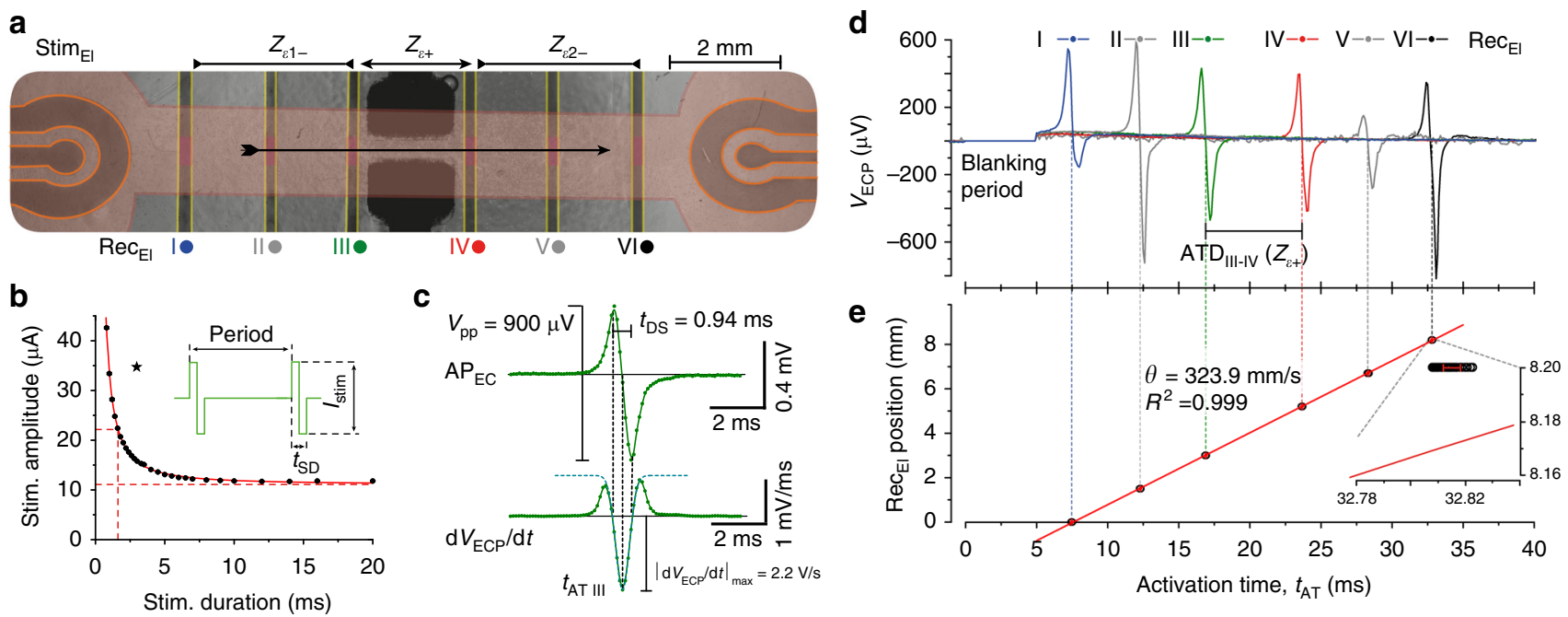

Fig. 3 Stimulation and recording with flexible gold ion-implanted electrodes. a Composite image of a cell strand (red) with stimulation (orange) and recording electrodes (yellow) on either side of the DEA electrodes (black). The center arrow illustrates the direction of action potential propagation. b Strength-duration curve for stimulation of the preparation with bi-polar current pulses (inset). The star indicates typical stimulation parameters used during the experiments. $\mathbf{c}$ Upper panel: $\mathrm{AP}_{\mathrm{EC}}$ as recorded by one electrode during propagated electrical activity along the cell strand. The $\mathrm{AP}_{\mathrm{EC}}$ exhibits an amplitude $\left(V_{\mathrm{pp}}\right)$ of $900 \mu \mathrm{V}$ and, as calculated from the Gaussian fit to the first derivative (lower panel), a downstroke time ( $\left.t_{\mathrm{DS}}\right)$ of $940 \mathrm{~ms}$ and a maximal downstroke velocity of $-2.2 \mathrm{~V} \mathrm{~s}^{-1}$. The negative peak of the fit was used to determine the local activation time $\left(t_{\mathrm{AT}}\right)$. $\mathbf{d} A \mathrm{P}_{\mathrm{ECs}}$ recorded by all electrodes during propagated electrical activity. The initial $5 \mathrm{~ms}$ of the signals containing the stimulation artifact were blanked. e Plot of local activation times vs. position for each recording electrode. Conduction velocity $\left(\theta=324 \mathrm{~mm} \mathrm{~s}^{-1}\right)$ is derived from the slope of a linear fit to the data. The insert indicates the presence of minimal $t_{\mathrm{AT}}$ variability (SD $<10 \mu \mathrm{s}, n=170$ serial stimulations) at ReC $\mathrm{El}_{\mathrm{EI}}$ III during 170 successive measurements 
strain protocol shown in Fig. 2c. The pacing rate permitted the determination of 25 activation time differences (ATDs) between pairs of specified $\mathrm{Rec}_{\mathrm{Els}}$ and, hence, between defined regions within the preparation during the initial and final baseline conditions and 12 determinations during each strain-relaxation cycle. Strain cycles were synchronized to pacing such that activation of the tissue occurred $300 \mathrm{~ms}$ after the onset of strain and relaxation, respectively, under static strain conditions. This allowed even the slowest ramps $\left(t_{\text {ramp }}=200 \mathrm{~ms}\right)$ to be completed before electrical activation. $\mathrm{AP}_{\mathrm{ECs}}$ recorded by the six $\mathrm{Rec}_{\mathrm{Els}}$ during action potential propagation under control (dashed line) and strained conditions (solid line) are shown in Fig. 4a. Strain $\left(V_{\varepsilon}=3.6 \mathrm{kV} ; t_{\text {ramp }}: 5 \mathrm{~ms}\right.$ ) caused an early appearance of $\mathrm{AP}_{\mathrm{ECs}}$ at $\mathrm{Rec}_{\mathrm{Els}}$ I-III and a delayed appearance at $\mathrm{Rec}_{\mathrm{Els}}$ IV-VI where the difference of the ATD recorded by $\mathrm{Rec}_{\mathrm{Els}}$ III and IV, straddling $Z_{\varepsilon+}$, increased from 6.851 to $7.004 \mathrm{~ms}(+2.7 \%)$. Strain profiles present in the $Z_{\varepsilon+}$ and $Z_{\varepsilon-}$ regions during these experiments are shown in Fig. $4 \mathrm{~b}$. In the $Z_{\varepsilon+}$ region, strain did not exactly mirror the actuation voltage $(3.6 \mathrm{kV}$ square pulses), but showed, as described above, an upward creep during DEA activation and a lesser pronounced downward creep during relaxation. An analogous behavior, albeit at reduced amplitudes and with reversed polarity, was observed in the $Z_{\varepsilon-}$ regions. As shown in Fig. 4c, ATDs measured between $\operatorname{Rec}_{\mathrm{Els}}$ III and IV closely followed the developed strain with ATDs increasing in the $Z_{\varepsilon+}$ region by $\sim 4 \%$ for the largest strain applied (10.0\%) while decreasing by $\sim-0.5 \%$ in the $Z_{\varepsilon-}$ region undergoing simultaneously compression by $\sim 1.4 \%$. This close correlation between strain and ATD is also evident during phases of creep that are mirrored by similarly shaped changes in ATDs. Discordance between strain and ATDs was present in the non-strained intervals where, following large strain amplitudes, the substrate showed near-complete relaxation, whereas ATDs exhibited a small but systematic increase following consecutive strain cycles (most notably for the first five cycles) and only slowly recovered during the final baseline recording period. (The $\mathrm{AP}_{\mathrm{EC}}$ recordings during strain application to the cell culture and the resulting modulation of the ATD are illustrated in the Supplementary Movie 1.) By contrast to the strain-dependent changes of ATDs, $t_{\mathrm{DS}}$ of signals recorded by $\mathrm{Rec}_{\mathrm{Els}}$ III and IV (Fig. 4d) were not affected by strain which suggests that active membrane properties and specifically the sodium inward current were little affected by the strain protocol used. The possibility that large electric fields associated with the activation of the DEAs may have affected conduction and, hence, ATDs, was investigated by mechanically stabilizing the substrate before applying standard strain protocols to the MaMEA. These measurements showed (Supplementary Fig. 14) that DEA activation in the absence of strain had no effect on ATDs, thereby excluding the presence of unspecific effects of electric fields on impulse conduction.

Effects of strain dynamics on impulse conduction. To investigate whether impulse conduction in strained preparations is dependent on strain rates and amplitudes, we systematically varied strain rates by altering $t_{\text {ramp }}$ from 5 to $200 \mathrm{~ms}$ for six strain amplitudes ranging from 0.2 to $10.0 \%$ in a single preparation. Strain-dependent modulation of ATD was determined in respect to the last ATD determination of the previous relaxation period to accommodate for creep effects. In accordance to the findings presented above (Fig. 4), ATDs in $Z_{\varepsilon+}$ increased with increasing strain amplitude while simultaneously decreasing in $Z_{\varepsilon-}$ (Fig. 5a).

Surprisingly, ATDs failed to show a dependence on strain rates even for the fastest rates $\left(18 \mathrm{~s}^{-1}\right)$ and largest strain amplitudes $(\sim 10.0 \%)$ tested. The same was observed for ATDs in the $Z_{\varepsilon-}$ regions which remained unaffected by changes in strain rates and amplitudes (Fig. 5a). When plotted as a function of strain amplitude (Fig. 5b), ATDs showed a linear dependence on strain with slopes $(\alpha)$ being virtually identical for the five different strain rates tested (see inset, $\alpha=0.302 \pm 0.008$; mean \pm SD). Compression zones $Z_{\varepsilon-}$ showed a highly similar behavior with reversed polarity and a slope of the linear fit amounting to 0.32 when including measurements from both the positive and negative strain zones. From the finding that ATDs are linearly dependent on strain, changes in true $\theta$ can be calculated by considering that $\frac{\theta}{\theta_{0}}=\frac{\varepsilon+1}{\alpha \varepsilon+1}$ (see Supplementary Note 9 for details). This function predicts a reduced dependence of $\theta$ on strain at large amplitudes which is indeed reflected by the data (Fig. 5c). Practically, the function indicates that $\theta$ increases by $\sim 7 \%$ for a strain amplitude of $10 \%\left(321-342 \mathrm{~mm} \mathrm{~s}^{-1}\right)$. Under the assumption that strain does not affect active membrane properties of the preparation, the increase of $\theta$ is explained by cable theory for the case that the ratio of axial resistance to gap junctional resistance is 0.29:0.71 (for details cf. Supplementary Note 10, the equivalent circuit diagram is illustrated in Supplementary Fig. 12, the scaling laws are derived in Supplementary Table 1). Experiments with five additional independent preparations being subjected to identical strain protocols produced highly similar results (cf. Supplementary Note 11 where five additional experiments are reproduced in Supplementary Fig. 13).

\section{Discussion}

With each pump cycle, the ventricles of the human heart are subject to positive strain during isovolumetric relaxation and during early and late diastolic filling which cause cardiomyocytes to be stretched by $\sim 13 \%$ at strain rates of $1-2 s^{-19,22-24}$. At comparable maximal strain amplitudes (10\%), the MaMEA presented here delivers strain rates surpassing physiological levels by an order of magnitude. The absolute precision of strain profiles reached $\sim 95 \%$ of the total strain amplitude due to the viscoelastic properties of the PDMS membrane, which caused an upward creep of strain during activation and a downward creep during deactivation of the DEAs with an opposite behavior observed in zones of tensile compression. By contrast to this short-term effects, mechanical long-term drift was minimal and repeated strain modulations had no lasting effects on the conduction characteristics as described in Supplementary Note 7. It was observed that the baseline remained within $0.3 \%$ of the initial recording. The gold ion-implanted compliant electrodes permitted continuous measurements of extracellular potentials during actuation of the substrate. The performance of these electrodes matched that of standard rigid MEA electrodes both in terms of stimulation efficiency and shape and size of the field potentials associated with the action potential upstroke ${ }^{25-27}$. Importantly, $\mathrm{AP}_{\mathrm{ECs}}$ recorded by compliant electrodes remained stable during experiments as illustrated by the finding that, after hundreds of strain cycles at maximal amplitude, $\mathrm{AP}_{\mathrm{EC}}$ characteristics remained unchanged. This finding, together with the observation that cell strands remained firmly attached to the substrate during the experiments, implies that cell adherence to the substrate was not compromised by high strain amplitudes and high strain rates. In parallel to the assessment of uniaxial stretch on conduction, the MaMEA has the unique feature of permitting the simultaneous investigation of the effect of compression on conduction. Apart from serving as an internal control that can unmask differential responses to positive and negative strain, the apposition of electronically coupled $Z_{\varepsilon+}$ and $Z_{\varepsilon+}$ regions may serve as a model for diseased hearts where zones of negative and positive strains coexist as is the case, for example, in infarct borderzones $^{28}$. Based on these particular properties of the DEA actuation, it is conceivable to construct geometrically defined strain patterns that reproduce specific patterns present in diseased 
a

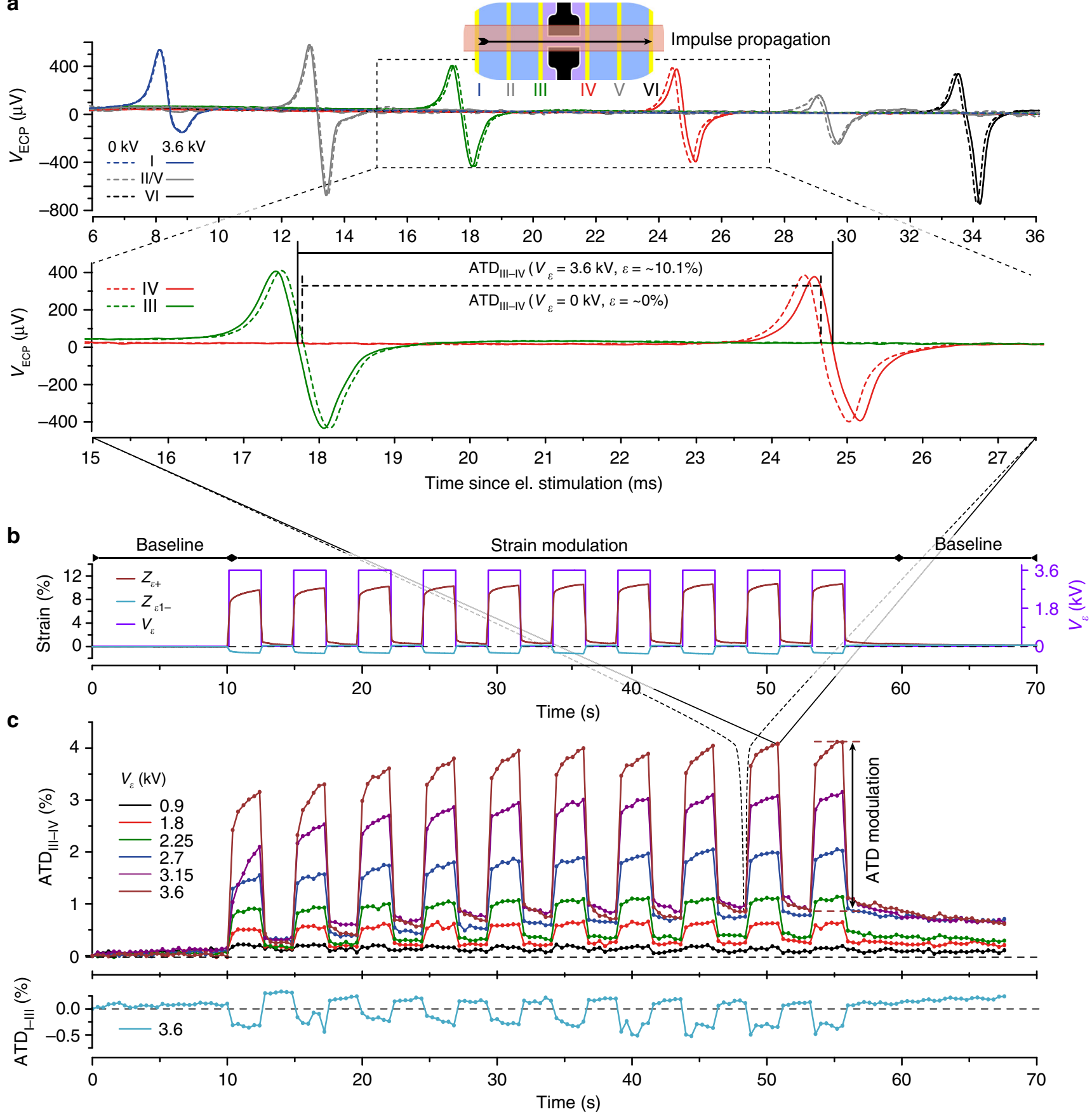

d

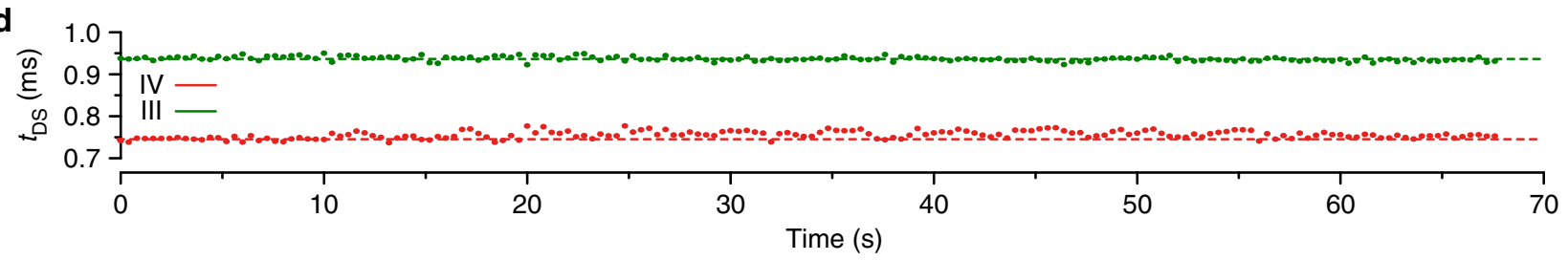

Fig. 4 Effect of strain amplitude on impulse conduction. a Upper panel: $\mathrm{AP}_{\mathrm{ECS}}$ recorded by electrodes I to VI during action potential propagation from left to right under control (dashed line) and strained conditions (solid line). Lower panel: Magnified view of the signals recorded by electrodes III and IV that straddle the positive strain zone show an increase of the activation time difference (ATD) by $2.7 \%$ during application of $10.1 \%$ strain. $\mathbf{b}$ Experimental strain protocol depicting the actuation voltages for the DEA (black) together with the measured response of the polydimethylsiloxane (PDMS) substrate in the positive (dark red) and negative strain (light blue) zones. c Modulation of ATD in the $Z_{\varepsilon+}$ region for different strain amplitudes ( $V_{\varepsilon}=1-4 \mathrm{kV}$ according to color scheme); ATD decrease in the $Z_{\varepsilon-}$ region is shown for $V_{\varepsilon}=3.6 \mathrm{kV}$ only (light blue). $\mathbf{d}$ Continuous determinations of downstroke times ( $t_{\mathrm{DS}}$ ) as recorded by electrodes III and IV at maximal strain amplitude $\left(V_{\varepsilon}=4 \mathrm{kV}\right)$. All data were obtained from a single preparation 


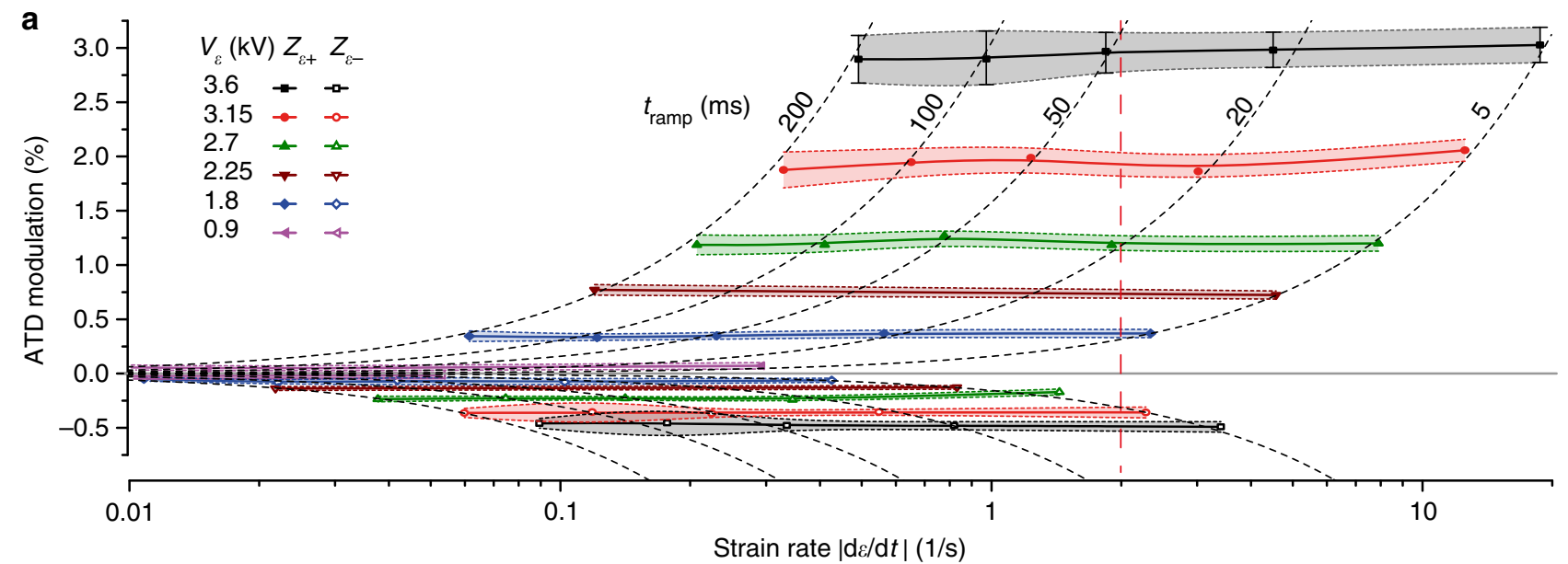

b

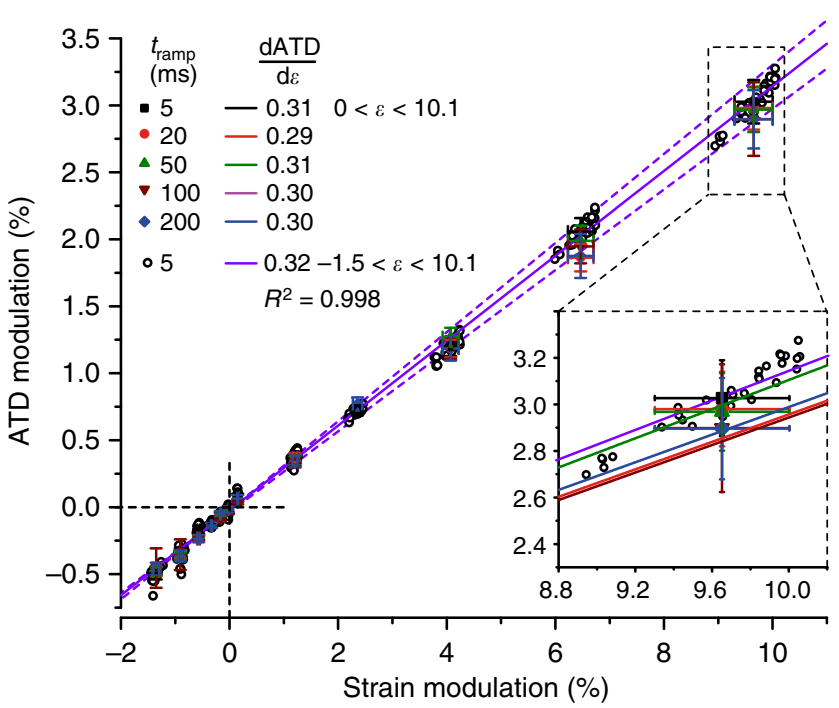

C

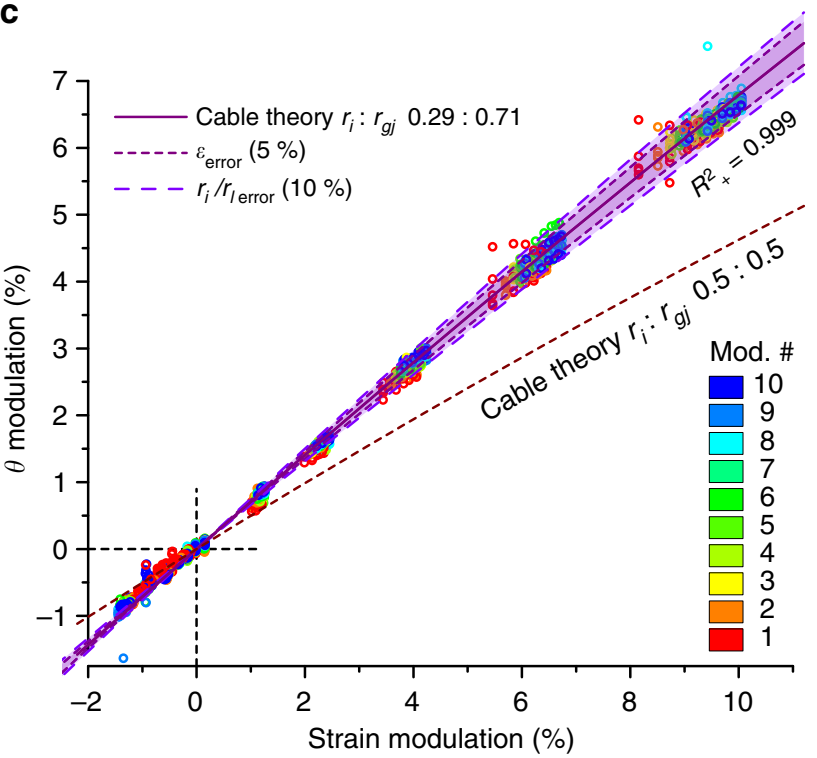

Fig. 5 Effects of increasing strain rates and strain amplitude on impulse conduction. a Modulation of activation time differences (ATDs) measured across the $Z_{\varepsilon+}$ region (above horizontal line) and $Z_{\varepsilon-}$ region (below horizontal line) as a function of maximal strain rates and amplitudes. The shaded area (extrapolated as a B-spline from the individual measurements) illustrates the extent of creep. The dotted lines depict identical ramp times (ATD $=\alpha \varepsilon t_{\text {ramp }}$ $\mathrm{d} \varepsilon / \mathrm{d} t$ ). b ATD modulation as a function of strain amplitude and strain rates. ATD modulation correlates linearly with strain amplitude (slope $\alpha=0.30$ $(\varepsilon>0)$ and $\alpha=0.32$ (all strain values)). The inset referring to the data obtained at largest strain amplitudes demonstrates that the slope is not dependent on strain rate $\left(\alpha_{\text {mean }}=0.302, \alpha_{\text {st.dev }}=0.008\right)$. Horizontal error bars denote variations of strain amplitudes over multiple modulations, vertical error bars denote variations of ATDs at given strain amplitudes caused by creep. c Dependence of the impulse conduction velocity, $\theta$, on strain amplitude. Circles denote results obtained during a complete set of experiments consisting of 10 successive strain cycles. All data were obtained from a single preparation. For further experiments cf. Supplementary Fig. 13. Source data are provided 35

hearts by appropriate redesign of the geometrical layout and number of active DEA electrodes ${ }^{10}$.

In the experiments presented, we fed the signals of the gold ion-implanted electrodes to the amplifiers using a fast time constant $\left(\tau_{c}\right)$ of the AC-coupling circuit. This has the advantage of stabilizing signal baselines without compromising the action potential upstroke related fast signals used for assessing straindependent changes in impulse conduction. On the downside, this setting causes suppression of slower signals as those associated with action potential repolarization. As shown in Supplementary Note 8, the MaMEA system is capable of recording repolarization related signals after appropriately increasing $\tau_{c}$. For the triangularly shaped rat action potential, the repolarization-dependent signal followed immediately upon the initial fast signal as reported before ${ }^{29}$. On the other hand, preparations consisting of stem-cell derived human cardiomyocytes exhibiting a pronounced plateau phase produced a repolarization signal that was separated from the initial fast signal by the length of the plateau $^{30,31}$. These findings demonstrate that the MaMEA system can be used with preparations different from rat ventricular cardiomyocytes and that, apart from investigating the effect of strain on conduction velocity as done in this study, the system may also be suited for the determination of the effects of dynamic strain cycles on action potential duration.

The coupling interval between completion of a given strain ramp and electrical activation in the experiments presented was systematically varied with the shortest interval amounting to $300 \mathrm{~ms}$. This interval can be deliberately shortened to $2-3 \mathrm{~ms}$, which corresponds to the time needed for the amplifiers to recover from saturation secondary to fast changing electrical fields during DEA activation/deactivation. Also, by using ramp times shorter than the ATD between the central two recording 
electrodes $(\sim 7 \mathrm{~ms})$, we found in preliminary experiments that it is feasible to assess effects of very fast strain events on conduction at the very moment when activation invades the central strained region of the MaMEA (data not shown). Such close coupling between strain events and electrical activation is not only essential for reproducing pathophysiological events like an impact to the chest interfering with ongoing electrical activation of the heart, but it is prerequisite for investigations of the effects of very fast inactivating stretch-sensitive ion channels like the piezo family ${ }^{32}$ on impulse conduction in excitable tissues.

The results of our measurements obtained at low strain rates concurred with findings of previous studies where cardiomyocyte cell strands had been subjected to $10.5 \%$ global static strain and where conduction was assessed using voltage sensitive dyes (ATD: $+3.2 \%$ vs. $+3.3 \%$; $\theta:+7.1$ vs. +7.0 ; maximal action potential upstroke velocities and $t_{\mathrm{DS}}$ not affected by strain $)^{33}$. The overall concordance of results obtained with the two different measurement techniques validates the MaMEA and demonstrates that measurements over $2.2 \mathrm{~mm}$ long strained regions (as opposed to $5 \mathrm{~mm}$ in the former study) are sufficient to obtain consistent results. Going beyond these findings, the present study showed that ATDs scale linearly with strain within the range of strain amplitudes tested. When calculating $\theta$ based on the actual lengths of the strained preparations divided by the respective ATDs, the resulting $\theta$ vs. strain relationship showed a $\frac{\theta}{\theta_{0}}=\frac{\varepsilon+1}{\alpha \varepsilon+1}$ dependence. Based on cable theory and as further described in the Supplementary Note 10, the relationship can be explained by strain-dependent changes of axial resistance under the assumption that cytoplasmic resistance contributes $29 \%$ and gap junctional resistance $71 \%$ to total axial resistance which is in accordance with recently published values obtained with the same type of preparation $(25 \%: 75 \%)^{3}$. Beyond these results, the MaMEA experiments demonstrated conclusively that conduction in strands of cardiomyocytes is insensitive to strain rates exceeding physiological values by an order of magnitude $\left(18 \mathrm{~s}^{-1}\right.$ at $10 \%$ strain amplitude). This surprising observation made in a total of six independent preparations (see Supplementary Note 11) suggests that cardiomyocytes can endure substantial mechanical stress without undergoing measurable electrophysiological changes at the cellular and multicellular level. The question then remains as to which mechanism(s) are responsible for heavy impact induced cardiac arrhythmias. Apart from the caveats (i) that the findings presented were obtained in vitro where MEC mechanisms may differ from those present in vivo, (ii) that conduction was determined $300 \mathrm{~ms}$ after abruptly increasing the strain, and (iii) that maximal strain amplitudes were possibly too moderate, the findings suggest that cells different from cardiomyocytes may be responsible for straindependent induction of cardiac arrhythmias with electronically coupled cardiac myofibroblasts being possible candidates ${ }^{33}$.

Given that the MaMEA is potentially able to generate strain rates reaching $1000 \mathrm{~s}^{-1} 10$ while simultaneously providing correlated strain amplitude modulation and electrophysiological recordings, the technology presented is ideally suited to systematically address fundamental questions regarding cellular mechanisms underlying MEC in the context of very fast strain events in excitable tissues.

\section{Methods}

Device fabrication. Sylgard 186 (Dow Corning) was casted as a sheet over a sacrificial polyacrylic acid layer. Target thickness was $80-125 \mu \mathrm{m}$. The sheet was mounted on a circular frame with a bi-axial pre-stretch of $1.2 \times 2.7$, resulting in a $25-\mu \mathrm{m}$-thick membrane as measured by optical interferometry. The addition of electrodes and the assembly of the culture wells is illustrated in the Supplementary Fig. 2. Carbon powder (Ketjenblack EC300; AkzoNobel) embedded in an elastomeric matrix was pad printed on both sides of the free-standing membrane. A

$\sim 2 \mu \mathrm{m}$ Silbione liquid silicone rubber (LSR 4305; Bluestar) isolation layer was pad printed to electrically insulate the strain electrodes from the culture medium and cells. The recording electrodes, $200 \mu \mathrm{m}$ wide, were gold ion implanted with $2.5 \mathrm{keV}^{11}$, and patterned using a steel shadow mask. A subsequent LSR 4305 layer was stamped to electrically insulate the $\mathrm{Rec}_{\mathrm{Els}}$ from the bath. Openings were included for cell contact. The patterned membranes were transferred to the PCB frames, electrical contact between the PDMS membrane and the PCB pads was ensured using SS-25 EMI/RFI conductive adhesive (Silicone Solutions). The DC resistance of the $\operatorname{Rec}_{\mathrm{Els}}$ channels ranged from $300 \Omega$ to $1.5 \mathrm{k} \Omega$ and their AC resistance (impedance) measured at $1 \mathrm{kHz}$ through the bath ranged from 1.7 to $9.6 \mathrm{k} \Omega$. DC and AC impedances changed $<6 \%$ for an applied strain of $10 \%$ in $Z_{\varepsilon+}$. Finally, the culture well was added to the device and sealed using LSR 4305. The cells were patterned using a Mylar mask. A Mylar backing immobilized the suspended membrane prior to the strain experiments and was left in place for the control experiments. Images of the electrodes and the cell strand are presented in Supplementary Figs. 5 and 6.

Device characterization. The DEA voltage-strain relation was measured using widefield microscopy. For calibration purposes, the devices were incubated with cell culture medium for $\geq 4$ days. Image processing (National Instruments vision assistant) was used for edge detection and strain calibration. A high-speed camera served to capture the strain response. The strain-voltage relation was tracked at $10 \mathrm{~Hz}$ frame rate while ramping the voltage linearly from 0 to $3.6 \mathrm{kV}$ over $30 \mathrm{~s}$ (Fig. 2a). The dynamic response was tracked at a frame rate of $1 \mathrm{kHz}$. The five ramp times were recorded over a total of $300 \mathrm{~ms}$ (Fig. 2b). The derivative of these data reflects the imposed strain rate. The corresponding voltage ramps are illustrated in Supplementary Fig. 4. The strain during the standard protocol (Fig. 2c) was calibrated optically at $100 \mathrm{~Hz}$ frame rate for each step function at multiple drive voltages.

Cell culture. Primary cultures of neonatal rat ventricular cardiomyocytes were obtained using established procedures and following Swiss guidelines for animal experimentation under the licencse BE27/17 of the state veterinary department of the canton Bern ${ }^{34}$. In brief, hearts from eight to ten neonatal rats (Wistar, 1-dayold) were excised, the ventricles were minced with scissors and the resulting small tissue pieces dissociated in Hank's balanced salt solution without $\mathrm{Ca}^{2+}$ and $\mathrm{Mg}^{2+}$ (BioConcept, 3-02F29-I) containing trypsin (0.1\%; Sigma, T4674) and pancreatin $(120 \mu \mathrm{g} / \mathrm{ml}$; Sigma, P3292). The dissociated cells were, after centrifugation, resuspended in medium M199 with Hank's salts (Sigma, M7653) supplemented with penicillin (20 U/ml; Sigma, P7794), vitamin $B_{12}(2 \mu \mathrm{g} / \mathrm{ml}$; Sigma, V2876), bromodexyuridine (100 $\mu \mathrm{mol} / \mathrm{L}$; Sigma, B-5002), vitamin C (18 $\mu \mathrm{mol} / \mathrm{L}$; Sigma, A-4544), epinephrine $(10 \mu \mathrm{mol} / \mathrm{L}$; Sigma, E4256), L-glutamine $(680 \mu \mathrm{M} / \mathrm{L}$; Sigma, G7513) and $10 \%$ neonatal calf serum (Biochrom, Bioswisstec, S0125). The cell suspension underwent differential preplating in cell culture flasks for $120 \mathrm{~min}$. The supernatant containing predominantly cardiomyocytes was collected, cell densities determined by with a hemocytometer, and cell density adjusted by dilution as to result in a seeding density of 3500 cardiomyocytes $/ \mathrm{mm}^{2}$. Before seeding, the MaMEA membrane was activated by ultaviolet-light exposure $(8 \mathrm{~min}$, UVO-Cleaner Model No. 342, Jelight Company Inc.) and subsequently coated with collagen type IV (C5533, Sigma). Patterned cell growth was realized using a lift-off technique. For this purpose, laser cut Mylar films that carried the negative of the desired cell pattern were affixed to the membrane prior to cell seeding. The cultures were kept in an incubator at $36^{\circ} \mathrm{C}$ in a humidified atmosphere containing $0.5 \% \mathrm{CO}_{2}$. Medium was exchanged $24 \mathrm{~h}$ post seeding with supplemented medium M199 (5\% serum) and every other day thereafter. Mylar masks were peeled off at the time of the first medium exchange resulting in well-defined strands of cardiomyocytes (see Sup plementary Note 5). Experiments were performed 72-96 h after seeding of the cardiomyocytes. hi-PSC-derived cardiomyocytes were obtained from Ncardia (Pluricyte ${ }^{\oplus}$ Cardiomyocyte Kit, Ncardia, Germany). Strand preparations were generated using the patterning procedures described for the rat cardiomyocytes above. Handling and culturing of the stem-cell-derived cardiomyocytes was done according to the detailed instructions given by the manufacturer. Experiments were performed with 4-day-old preparations.

Experiments. All experiments were performed in an incubator set to $36^{\circ} \mathrm{C} / 0.5 \%$ $\mathrm{CO}_{2}$. During experiments lasting $\leq 3 \mathrm{~h}$, the MaMEA wells were covered by a lid. The MaMEA was mounted to the motherboard that was plugged into a DAQ $(6 \mu \mathrm{V}$ resolution, $4 \mathrm{mV}$ dynamic range) residing in the incubator. After assembly in the incubator, the system was left to stabilize for 30-60 min. Experiments were started once the drift in ATDs became negligible. To be included in experiments, the following criteria had to be met by the preparations: (i) $\geq 5$ recording electrodes were operational, (ii) the DEA actuator was able to generate $\geq 10 \%$ strain without breakdown, and (iii) cell strands displayed a uniform structure and displayed continuous conduction under control conditions.

High-voltage drive and data recording. The high-voltage signal $\left(V_{\varepsilon}\right)$ was gen erated by an arbitrary function generator (Agilent 33521A) and amplified by a factor of 500x using an HVA series amplifier (Ultravolt) with a bi-polar output of

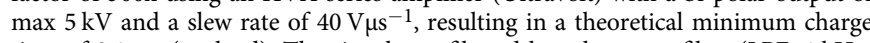
time of $0.1 \mathrm{~ms}$ (no load). The signal was filtered by a low-pass filter (LPF, $1 \mathrm{kHz}$ cutoff, resulting in a minimum $5 \mathrm{~ms}$ rise time). The applied voltage was monitored using an oscilloscope. In addition to step functions, the DEA voltage was also 
ramped as $\sim \sqrt[3]{\frac{t}{t_{0}}}$, resulting in a linear strain ramp exhibiting a pre-determined ramp rate. Only the standard protocol strain profiles were imposed on the cell strand during measurements.

Electrical and mechanical stimulation protocol. Preparations were stimulated at double threshold amplitude with a biphasic current pulse generated by a custommade stimulator. For each data set, the cell strand was stimulated for $30 \mathrm{~s}(20 \mathrm{~s}$ prestimulation, $10 \mathrm{~s}$ recorded) before switching on the DEA voltage (100 $\mathrm{ms}$ after the last activation of the preparation) imposing tensile and compressive strain on the preparation. The standard protocol was based on 10 strain cycles at $4.8 \mathrm{~s}$ period and a $50 \%$ duty cycle. The amplitude of the DEA voltage ranged from 1 to $3.6 \mathrm{kV}$ and the ramp time was set to $5,20,50,100$, or $200 \mathrm{~ms}$. For each data set, the slowest and smallest amplitude was tested first ( $200 \mathrm{~ms}$ rise time to $0.9 \mathrm{kV}$ ) followed by increasing the amplitude at constant ramp times. The data set was concluded with the $5 \mathrm{~ms}$ ramp sequence to $3.6 \mathrm{kV}$ that was followed by a 10-s-long recording of stimulated $\mathrm{AP}_{\mathrm{EC}}$ under non-strained conditions. The overall measurement protocol lasted $10+48+10 \mathrm{~s}=68 \mathrm{~s}$ during which each electrode recorded $170 \mathrm{AP}_{\mathrm{ECs}}$.

In all experiments, an initial $68 \mathrm{~s}$ of baseline recording was performed (electrical but no mechanical stimulation) that was used to characterize basic

electrophysiological properties of the strand. Performance parameters and stability are illustrated in Supplementary Figs. 8 and 9.

AP $P_{E C}$ analysis. Electrograms were analyzed using MATLAB following the procedures described in Supplementary Note 6. Data were blanked, that is, set to background level, during the electrical stimulation artifacts. Similarly, during the $V_{\varepsilon}$ ramp causing the mechanical stimulation, capacitive coupling resulting in electrical noise prohibiting recording of APs and, consequently, the period of DEA modulation was blanked as well. The shortest blanking could be as brief as $10 \mathrm{~ms}$, but always needed to exceed the ramp time. Given that the first AP was electrically triggered $300 \mathrm{~ms}$ after the mechanical stimulation, the $V_{\varepsilon}$ blanking did not interfere with the recording of electrograms (for details see Supplementary Fig. 7).

Detailed procedures regarding the data evaluation protocol are described in Supplementary Note 6. Electrical noise levels are illustrated in Supplementary Fig. 7, and an example of stability and timing accuracy during a baseline recoding is presented in Supplementary Fig. 8.

A control experiment was performed to determine the effect of the DEA voltage in the absence of mechanical strain. For this purpose, the membrane was immobilized by leaving the Mylar on the substrate while actuating the DEA up to $3.5 \mathrm{kV}$. Results are depicted in Supplementary Fig. 14 (Supplementary Note 12), with the corresponding $p$ value indicating that control values were not different from immobilized values $(p=0.31)$ while, as expected, ATD changed significantly for both strain zones after removal of Mylar. This corroborates previous findings with DEA actuators where, in the presence of an immobilized membrane, electrical fields had no measurable effect on cell cultures ${ }^{15}$.

To exclude that HV, capacitively coupled to the cultured cells, could cause adverse effects on cell viability, cardiomyocytes were grown on an array of metallic electrodes covered with $100 \mu \mathrm{m}$ of PDMS and were exposed to a modulating highvoltage signal of $4 \mathrm{kV}$ at $1 \mathrm{~Hz}$ over $6 \mathrm{~h}$. A cell viability kit (Biotium \#30065) revealed no differences in the density of apoptotic and necrotic cells when compared to control samples (the data are presented in the Supplementary Fig. 16, a discussion on this topic can be found in Supplementary Note 13, including a graphical illustration of electric field strengths found in biological systems presented in Supplementary Fig. 15).

Ethical compliance. The experiments are compliant and were performed following strict federal regulations under license BE27/17 of the State Veterinary Department of the Canton of Bern.

Reporting summary. Further information on experimental design is available in the Nature Research Reporting Summary linked to this article.

\section{Data availability}

Source data underlying Fig. 5 and Supplementary Fig. 13 are provided as a Source Data file (https://doi.org/10.5281/zenodo.2541654). All other relevant data are available from the authors.

Received: 11 June 2018 Accepted: 24 January 2019

Published online: 19 February 2019

\section{References}

1. Orini, M. et al. Mechano-electrical feedback in the clinical setting: current perspectives. Prog. Biophys. Mol. Biol. 130, 365-375 (2017).

2. Kléber, A. G. \& Rudy, Y. Basic mechanisms of cardiac impulse propagation and associated arrhythmias. Physiol. Rev. 84, 431-488 (2004)
3. Buccarello, A., Azzarito, M., Michoud, F., Lacour, S. P. \& Kucera, J. P. Uniaxial strain of cultured mouse and rat cardiomyocyte strands slows conduction more when its axis is parallel to impulse propagation than when it is perpendicular. Acta Physiol. https://doi.org/10.1111/apha.13026, 1-19 (2018).

4. Kang, W. H. et al. Alterations in hippocampal network activity after in vitro traumatic brain injury. J. Neurotrauma 32, 1011-1019 (2015).

5. Schürmann, S. et al. The IsoStretcher: an isotropic cell stretch device to study mechanical biosensor pathways in living cells. Biosens. Bioelectron. 81, 363-372 (2016).

6. Friedrich, O. et al. Adding dimension to cellular mechanotransduction: advances in biomedical engineering of multiaxial cell-stretch systems and their application to cardiovascular biomechanics and mechano-signaling. Prog. Biophys. Mol. Biol. 130, 170-191 (2017).

7. Michielin, F., Serena, E., Pavan, P. \& Elvassore, N. Microfluidic-assisted cyclic mechanical stimulation affects cellular membrane integrity in a human muscular dystrophy in vitro model. RSC Adv. 5, 98429-98439 (2015).

8. Kim, D.-H., Wong, P. K., Park, J., Levchenko, A. \& Sun, Y. Microengineered platforms for cell mechanobiology. Annu. Rev. Biomed. Eng. 11, 203-233 (2009).

9. Kobirumaki-Shimozawa, F. et al. Nano-imaging of the beating mouse heart in vivo: importance of sarcomere dynamics, as opposed to sarcomere length per se, in the regulation of cardiac function. J. Gen. Physiol. 147, 53-62 (2016)

10. Poulin, A. et al. An ultra-fast mechanically active cell culture substrate. Sci. Rep. 8, 9895 (2018).

11. Rosset, S., Niklaus, M., Dubois, P. \& Shea, H. R. Metal ion implantation for the fabrication of stretchable electrodes on elastomers. Adv. Funct. Mater. 19, 470-478 (2009).

12. Pelrine, R., Kornbluh, R., Pei, Q. \& Joseph, J. High-speed electrically actuated elastomers with strain greater than $100 \%$. Science (80-) 287, 836-839 (2000).

13. Rosset, S. \& Shea, H. R. Small, fast, and tough: shrinking down integrated elastomer transducers. Appl. Phys. Rev. 3, 031105 (2016).

14. Anderson, I. A., Gisby, T. A., McKay, T. G., O’Brien, B. M. \& Calius, E. P. Multi-functional dielectric elastomer artificial muscles for soft and smart machines. J. Appl. Phys. 112, 041101 (2012).

15. Poulin, A., Saygili Demir, C., Rosset, S., Petrova, T. V. \& Shea, H. Dielectric elastomer actuator for mechanical loading of 2D cell cultures. Lab Chip 16, 3788-3794 (2016).

16. Akbari, S., Rosset, S. \& Shea, H. R. Improved electromechanical behavior in castable dielectric elastomer actuators. Appl. Phys. Lett. 102, 071906 (2013).

17. Pelrine, R. E., Kornbluh, R. D. \& Joseph, J. P. Electrostriction of polymer dielectrics with compliant electrodes as a means of actuation. Sens. Actuators A 64, 77-85 (1998)

18. Geddes, D. M., Cargill, R. S., LaPlaca, M. C., Cargill Ii, R. S. \& LaPlaca, M. C Mechanical stretch to neurons results in a strain rate and magnitudedependent increase in plasma membrane permeability. J. Neurotrauma 20 , 1039-1049 (2003)

19. Bain, A. C. \& Meaney, D. F. Tissue-level thresholds for axonal damage in an experimental model of central nervous system white matter injury. J. Biomech. Eng. 122, 615 (2000).

20. Bayly, P. V., Black, E. E., Pedersen, R. C., Leister, E. P. \& Genin, G. M. In vivo imaging of rapid deformation and strain in an animal model of traumatic brain injury. J. Biomech. 39, 1086-1095 (2006).

21. Ask, A., Menzel, A. \& Ristinmaa, M. Modelling of viscoelastic dielectric elastomers with deformation dependent electric properties. Procedia IUTAM 12, 134-144 (2015).

22. Kasner, M. et al. Global strain rate imaging for the estimation of diastolic function in HFNEF compared with pressure-volume loop analysis. Eur. J. Echocardiogr. 11, 743-751 (2010).

23. Wang, J., Khoury, D. S., Thohan, V., Torre-Amione, G. \& Nagueh, S. F. Global diastolic strain rate for the assessment of left ventricular relaxation and filling pressures. Circulation 115, 1376-1383 (2007).

24. Sonnenblick, E. H., Ross, J., Covell, J. W., Spotnitz, H. M. \& Spiro, D. The ultrastructure of the heart in systole and diastole. Changes in sarcomere length. Circ. Res. 21, 423-431 (1967).

25. Kucera, J. P., Heuschkel, M. O., Renaud, P. \& Rohr, S. Power-law behavior of beat-rate variability in monolayer cultures of neonatal rat ventricular myocytes. Circ. Res. 86, 1140-1145 (2000).

26. Kondratyev, A. A., Ponard, J. G. C., Munteanu, A., Rohr, S. \& Kucera, J. P. Dynamic changes of cardiac conduction during rapid pacing. Am. J. Physiol. Circ. Physiol. 292, H1796-H1811 (2007).

27. Prudat, Y. \& Kucera, J. P. Nonlinear behaviour of conduction and block in cardiac tissue with heterogeneous expression of connexin 43. J. Mol. Cell. Cardiol. 76, 46-54 (2014).

28. Wenk, J. F. et al. Regional left ventricular myocardial contractility and stress in a finite element model of posterobasal myocardial infarction. J. Biomech. Eng. 133, 044501 (2011). 
29. Uchida, T., Kitora, R. \& Gohara, K. Temperature dependence of synchronized beating of cultured neonatal rat heart-cell networks with increasing age measured by multi-electrode arrays. Res Artic. Trends Med. 18, 1-10 (2018).

30. Asakura, K. et al. Improvement of acquisition and analysis methods in multielectrode array experiments with iPS cell-derived cardiomyocytes. $J$. Pharmacol. Toxicol. Methods 75, 17-26 (2015).

31. Tertoolen, L. G. J., Braam, S. R., van Meer, B. J., Passier, R. \& Mummery, C. L. Interpretation of field potentials measured on a multi electrode array in pharmacological toxicity screening on primary and human pluripotent stem cell-derived cardiomyocytes. Biochem. Biophys. Res. Commun. 497, 1135-1141 (2018).

32. Wu, J., Lewis, A. H. \& Grandl, J. Touch, tension, and transduction-the function and regulation of piezo ion channels. Trends Biochem. Sci. 42, 57-71 (2017).

33. Grand, T., Salvarani, N., Jousset, F. \& Rohr, S. Aggravation of cardiac myofibroblast arrhythmogeneicity by mechanical stress. Cardiovasc. Res. 104, 489-500 (2014).

34. Rohr, S., Flückiger-Labrada, R. \& Kucera, J. P. Photolithographically defined deposition of attachment factors as a versatile method for patterning the growth of different cell types in culture. Pflug. Arch. Eur. J. Physiol. 446, 125-132 (2003).

35. Imboden, M. et al. Source data underlying Fig. 5 and Supplementary Fig. 13. https://doi.org/10.5281/zenodo.2541654 (2018).

\section{Acknowledgements}

This work was supported by the Marie Skłodowska-Curie Action H2020-MSCA-IF-2015 \#701614 (to M.I.) and the Swiss National Science Foundation grant CR32I3_166326 and 20020_165993 (to H.S.) and 310030_169234 (to St.R.) We would like to thank Olexandr Gudozhnik for his assistance and advice on the motherboard layout and Regula Flückiger Labrada for her tireless cell culture work and device preparation.

\section{Author contributions}

St.R. and H.S. conceived the experiments. M.I. and A.P. designed the DEA. Sa.R., H.S. and A.P. advised on the design and fabrication of the device and S.R. developed the gold ion implantation method. M.I. designed the $\mathrm{Rec}_{\mathrm{Els}}$ and $\mathrm{Stim}_{\mathrm{El}}$, $\mathrm{PCB}$ frames, and modular holder. M.I. fabricated the devices. M.I. and E.C. performed the experiments, which was overseen by H.S. and St.R. C.D. designed and build the DAQ. M.I. analyzed the data. M. I., E.C. and St.R. interpreted the data. M.I. and St.R. wrote the manuscript. All authors read, edited, and discussed the manuscript and agree with the claims made in this work.

\section{Additional information}

Supplementary Information accompanies this paper at https://doi.org/10.1038/s41467019-08757-2.

Competing interests: The authors declare no competing interests.

Reprints and permission information is available online at http://npg.nature.com/ reprintsandpermissions/

Journal peer review information: Nature Communications thanks Luca Sala and Janos Voros for their contribution to the peer review of this work.

Publisher's note: Springer Nature remains neutral with regard to jurisdictional claims in published maps and institutional affiliations.

(c) Open Access This article is licensed under a Creative Commons Attribution 4.0 International License, which permits use, sharing, adaptation, distribution and reproduction in any medium or format, as long as you give appropriate credit to the original author(s) and the source, provide a link to the Creative Commons license, and indicate if changes were made. The images or other third party material in this article are included in the article's Creative Commons license, unles indicated otherwise in a credit line to the material. If material is not included in the article's Creative Commons license and your intended use is not permitted by statutory regulation or exceeds the permitted use, you will need to obtain permission directly from the copyright holder. To view a copy of this license, visit http://creativecommons.org/ licenses/by/4.0/.

(C) The Author(s) 2019 